\title{
Introduction to Part II: Inhabiting
}

\author{
Thomas Kvan and Kenn Fisher
}

\begin{abstract}
This section presents research in inhabiting new learning environments. The topic is introduced, and a brief review is given for each chapter in the following section.
\end{abstract}

This section brings together papers that have a particular focus on the ways in which the users inhabit the spaces. By reflecting on their use of the space, their movements and the learning activities, insights are offered on the ways we can support better learning through physical interventions and through changes in mindsets.

Broens argues that physical diversity within a larger space is more beneficial for teacher collaboration than the flexibility of the furniture or the architecture. Drawing on her background in educational anthropology and many years of experience teaching young children, she focuses on the mobility of the inhabitants rather than the effectiveness of the material affordances in the ILE's. Broens observed a team of teachers for 300 hours, with associated interviews using an ethnographic approach, to understand their spatial behaviours.

The study found that teacher mindsets often saw them default to a traditional classroom approach, but that 'open' flexible innovative learning spaces offered greater agency for teachers to interact with each other, without built barriers impeding in this activity. She proposes that it is the social relations established within a space rather than the physical elements of the space that are essential, yet these cannot exist without the physical. Teaching only under the sky is not the option and, even then, the physical is contributing. She notes, however, that certain spaces enabled or promoted more active engagement in the experience of teaching and learning. This in turn supported better collaboration. She concludes that an intentional diversity of spaces is more effective in supporting collaboration than the provision of flexibility. The need, however, is for the users to adapt themselves to the opportunity. As she

T. Kvan $(\bowtie) \cdot$ K. Fisher

The University of Melbourne, Melbourne, VIC, Australia

e-mail: tkvan@unimelb.edu.au

K. Fisher

e-mail: fisherk@unimelb.edu.au 
notes, 'the use of space is a relational construct not only between the teachers, and the space, but also between the teachers and the students and between the students themselves'.

Morrison and Healy explore how research into learning environments might itself be conducted differently. Starting with a performative art experiment, they develop a perspective on the role of the researcher, the focus of their research and the methods by which they participate, in particular through presence. Their observation is that the researcher is a present actor in the research, a collaborator in the school environment in their gathering and interpretation of the data. In the context of inhabiting, this observation is critical, reflecting the participative process of inhabitation. Users of spaces do not take on the designer's prescribed intent, they co-design and redesign the space as it is used.

Shapiro proposes a complementary perspective that introduces a method to describe and interpret the spatial interaction of people over time, an approach called interaction geography. By examining the engagement of space in a museum, mapping movement and conversation throughout a visit, and visualising this in a multi-modal mapped representation, a spatial representation of learning is developed. Specifically, he reviews the Mondrian Transcription approach which tracks movement and conversation in space and time and then deploys the Interaction Geography Slicer (IGS) to illustrate these patterns in a dynamic visualisation analysis. The resulting diagram of the dynamic engagement in space while in a learning activity offers us a broader perspective of the group activity that may enable us to reconsider spatial support of pedagogy. Shapiro concludes that this approach is highly interdisciplinary, and can be scaled, but it also needs some additional technical development to interpret the results that vary with scale.

If one of the goals of ILEs is to facilitate better participation in learning, the ease of communication is a key factor. A goal of ILEs is to ensure participation by students is broad and equal, so spatial arrangements do not prejudice or privilege anyone in the space. A common observation of ILEs is that there are fewer acoustical divisions and hence an activity in one area can readily impinge on other users and their activities.

Rose-Munro focusses on a particular aspect of spatial interaction in learning, the auditory. In particular, she brings our attention to the support of learning by all, including those with constraints with hearing abilities, by considering speaking and listening. She notes that $7-10 \%$ of students are likely to have 'suboptimal hearing abilities', and also points to the increase in students having autism spectrum disorders who will benefit from reduced auditory distractions. The connected visual spaces of ILEs bring with them interconnected auditory environments; while sight may be limited by interposing temporary barriers such as paper or screens, sound is not so easily contained. The transit of sound is less easily intuited and thus more difficult to anticipate and manage. Supporting active listening for students with hearing problems is more challenging than reducing visual distractions.

Rose-Munro found that students intuitively knew the 'sweet acoustical spots' in various learning spaces and inevitably settled in those spaces. But she also found that sound loop affordances for teachers also provided much greater clarity for students. She also found that student engagement was related to student agency 
and the formation of trust with teachers. With these findings, she helps us understand how to improve ILEs as inclusive spaces for such students, for example by developing acoustic nooks and by trusting peers to mitigate the difficulties through personal interaction.

Thomas Kvan (Australia) is recognised for his pioneering work in design, digital environments and design management and has held senior leadership roles in several universities as Dean and Pro Vice Chancellor. He was the founding co-Director of LEaRN (the Learning Environments Applied Research Network) delivering multidisciplinary research on learning and architecture, and was founding Director of AURIN (the Australian Urban Research Information Network) that developed a national digital infrastructure, both networks hosted at The University of Melbourne. He has published over 180 publications in academic, professional and popular channels. He is currently founding Dean of the School of Design at the South University of Science and Technology (SUSTech) in China.

Kenn Fisher (Australia) is recognised as one of the leading learning environment specialists practising locally, nationally and internationally for over three decades. He has practised in Australia, Asia, the Middle East and Europe and as a consultant to the OECD (where he held the post of Head of the Programme on Educational Building in Paris in 1997/8) and UNESCO. He is multiskilled in a range of disciplines having practised in all education sectors as a teacher and academic, a strategic facility and campus planner and as a project, facility and design manager. He has been engaged by more than universities worldwide, over a dozen vocational training and community college clients, a number of State and National Government Ministries of Education, many school organisations and Government and corporate entities. Kenn is an Associate Professor in Learning Environments at The University of Melbourne's School of Design (MSD).

Open Access This chapter is licensed under the terms of the Creative Commons Attribution 4.0 International License (http://creativecommons.org/licenses/by/4.0/), which permits use, sharing, adaptation, distribution and reproduction in any medium or format, as long as you give appropriate credit to the original author(s) and the source, provide a link to the Creative Commons license and indicate if changes were made.

The images or other third party material in this chapter are included in the chapter's Creative Commons license, unless indicated otherwise in a credit line to the material. If material is not included in the chapter's Creative Commons license and your intended use is not permitted by statutory regulation or exceeds the permitted use, you will need to obtain permission directly from the copyright holder.

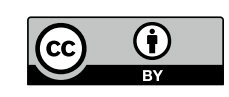

